\title{
TAXONOMY OF MEDICAL DEVICES IN THE LOGIC OF HEALTH TECHNOLOGY ASSESSMENT
}

Cornelia Henschke

Department of Health Care Management, Technische Universität Berlin

cornelia.henschke.1@tu-berlin.de

Dimitra Panteli

Department of Health Care Management, Technische Universiẗ̈t Berlin

\author{
Matthias Perleth \\ Medical Consultancy Department, Federal Joint Committee \\ Reinhard Busse \\ Department of Health Care Management, Technische Universität Berlin
}

Objectives: The suitability of general HTA methodology for medical devices is gaining interest as a topic of scientific discourse. Given the broad range of medical devices, there might be differences between groups of devices that impact both the necessity and the methods of their assessment. Our aim is to develop a taxonomy that provides researchers and policy makers with an orientation tool on how to approach the assessment of different types of medical devices.

Methods: Several classifications for medical devices based on varying rationales for different regulatory and reporting purposes were analyzed in detail to develop a comprehensive taxonomic model.

Results: The taxonomy is based on relevant aspects of existing classification schemes incorporating elements of risk and functionality. Its $9 \times 6$ matrix distinguishes between the diagnostic or therapeutic nature of devices and considers whether the medical device is directly used by patients, constitutes part of a specific procedure, or can be used for a variety of procedures. We considered the relevance of different device categories in regard to HTA to be considerably variable, ranging from high to low.

Conclusions: Existing medical device classifications cannot be used for HTA as they are based on different underlying logics. The developed taxonomy combines different device classification schemes used for different purposes. It aims at providing decision makers with a tool enabling them to consider device characteristics in detail across more than one dimension. The placement of device groups in the matrix can provide decision support on the necessity of conducting a full HTA.

Keywords: Health technology assessment, Medical devices, Classification

Health technologies such as medical devices are an essential part of health care. The European Union defines a medical device as " $[\ldots]$ any instrument, apparatus, appliance, software, material or other article, whether used alone or in combination, together with any accessories [...]" (1), which includes a broad range of different products for purposes such as prevention, diagnosis, monitoring, treatment, alleviation of disease, or compensation for an injury or handicap (1). Many of these devices have led to important improvements in diagnosis and treatment of diseases such as better health outcomes in terms of higher life expectancy and/or better quality of life (2-4). However, their rapid pace of innovation might also be associated with risks (5) and cause harm to patients. Recent examples arising from the use of medical devices include recalls of specific types of breast implants (6), artificial hips (7), and implantable cardioverter-defibrillators (8). Furthermore, fast-paced innovation is accompanied by rising health expenditures $(9 ; 10)$. The combined imperatives of patient benefit and safety as well as cost containment have led many countries to introduce regulatory instruments to identify technologies that minimize risk and ensure value and value for money.
There are two approaches to regulate medical technologies depending on the point in time when regulation applies relative to market authorization. The first approach concerns premarket evaluations, which cover safety and performance aspects. An additional demonstration of efficacy can be required depending on the authorization system and the risk of the device (e.g., high risk devices in the United States that are regulated by the Food and Drug Administration [FDA]) $(11 ; 12)$. The second approach encompasses postmarket evaluation systems that additionally aim to consider effectiveness in the usage context, cost-effectiveness and other domains potentially relevant for coverage decision making, such as legal, ethical, socio-cultural, and organizational consequences (13). Health technology assessment (HTA) falls under this second approach and aims at informing regulatory decision makers once marketing authorization has been granted. It is applied to a broad range of technologies, including pharmaceuticals, medical devices, and public health interventions. The final intention of HTA is to provide the evidence base for decision makers, primarily at health system level, on the consequences of adopting a given technology across the aforementioned domains $(14 ; 15)$. However, most formal decision structures incorporating evidence-based 
approaches were established for pharmaceuticals (16), which also have been the main focus of guidelines for economic evaluation (17). The relevance of general methodology for medical devices, both in HTA as a whole and for economic evaluation in particular, has been gaining interest as a topic of scientific discourse, especially in light of new regulatory provisions for their market authorization (18).

There are particularities characterizing medical devices, which merit differentiated assessment practices ranging from trial design to statistical methods to be used for the analysis of clinical and economic data (19-21). Assessors of medical devices, especially if they are provided as part of invasive and highrisk diagnostic and therapeutic procedures frequently face the problem of a lack of randomized controlled trials (RCTs), which partly derives from lower evidence hurdles in market authorization processes compared with pharmaceuticals $(17 ; 22 ; 23)$. McCulloch et al. (21) describe particularities and difficulties of evaluations in the field of surgical innovations, and propose possible solutions for the assessment of effectiveness against current standards. They give options for alternative study designs, such as parallel group nonrandomized studies or controlled interrupted-time series studies, to be used when randomized trials are not feasible for ethical or pragmatic reasons (21;24).

Furthermore, given the large, heterogeneous spectrum of devices used for diagnostic and/or therapeutic purposes, it is safe to assume that there might be device-specific characteristics that should be accounted for in HTA practice. For example, the evaluation of diagnostic devices might be complicated by difficulties in separating the value of the diagnosis itself from the value of improved outcomes due to subsequent treatment $(17 ; 25)$. Furthermore, many medical devices are subject to device-operator interaction, which makes the efficacy of the device contingent on the learning curve of the operator $(17 ; 22 ; 24)$. Finally, the implementation of a new device might be associated with wider organizational and economic implications for providers, such as the need for professional trainings or new infrastructures and associated investments (17). These particularities complicate the debate about what the related health benefits are, where and to whom these accrue and how rational decisions regarding coverage in relation to effectiveness and cost can be made.

Medical devices have been classified in different ways. While classifications facilitating market authorization aim at categorizing medical devices according to the degree of risk assigned to them, procurement classifications adopt a perspective relevant to pricing and reimbursement decisions. To our knowledge, as of yet there is no detailed classification that looks at medical devices from the viewpoint of HTA. Therefore, the aim of this study is (i) to clarify, describe and distinguish different categories of medical devices in the context of HTA for decision making on value and coverage and (ii) to develop a taxonomic model that incorporates elements of exist- ing classification schemes. With the ultimate goal of providing researchers and policy makers with an orientation guide on how to approach the assessment of medical devices, the taxonomy aims at (i) highlighting the extent to which HTA is necessary for different device categories and (ii) indicating if and how assessment tools should be tailored to include device-specific elements and achieve best results depending on different categories of medical devices. As a first step, the model focuses on Europe and does not account for differences in pre- and postmarket approval processes of medical devices beyond the European Union (EU) context.

\section{METHODS}

The research centered on different classification schemes for medical devices. To identify several classifications and nomenclatures for medical devices based on varying rationales for different regulatory and reporting purposes, selected to account for different characteristics of medical devices, we carried out a search of literature. We first carried out a PubMed search to identify relevant published literature and only considered studies with a focus on classification and nomenclatures of medical devices. We also reviewed legislative and policy documents in the European context discussed in the included studies. These included the current European Union regulatory framework for medical devices consisting of three directives used for the licensing process, the international classification for medical devices used by the Global Medical Device Nomenclature (GMDN), and the OECD Classification of Health Care Functions, which considers, inter alia, reimbursement aspects. These frameworks were analyzed in detail to inform the development of a comprehensive taxonomic model.

\section{RESULTS}

\section{Background on Existing Classifications of Medical Devices}

Classification of Medical Devices According to EU Directives. The current European Union regulatory framework for medical devices consists of three directives: Council Directive 93/42/EEC on medical devices (MDD), Council Directive 90/385/EEC on active implantable medical devices (AIMDD) and Council Directive 98/79/EC on in vitro diagnostic medical devices (IVDD) (2628). Medical device manufacturers are allowed to market their products across all EU member states after receiving a Conformité Européenne (CE) mark, which demonstrates that the safety criteria, quality in terms of reliability, and performance are fulfilled $(11 ; 29)$. However, these regulations do not require manufacturers to demonstrate the effectiveness or cost-effectiveness of their products to obtain marketing authorization (30).

MDD is applicable to all medical devices that are neither an active implantable medical device nor an in vitro diagnostic medical device. During conformity assessment, medical devices are categorized in one of four classes (I, IIa, IIb, or 
III) according to the potential risk associated with their use. This Directive contains eighteen rules that guide categorization based on device characteristics such as nonactive versus active devices, noninvasive versus invasive devices and short-term use versus long-term use of invasive devices. Different conformity assessment procedures have to be implemented depending on device class (26).

AIMDD is valid for active implantable medical devices, which rely on sources such as electrical energy and are "[ $[.$. intended to be totally or partially introduced, surgically or medically, into the human body or by medical intervention into a natural orifice, and which is intended to remain after the procedure $[\ldots]$ ". In contrast to MDD, AIMDD contains no differentiation according to identified risks associated with the device. However, it does distinguish between custom-made products developed specifically for an individual patient, and products intended for clinical investigations, which include active implantable medical devices used only by specialist doctors when conducting investigations in a clinical environment. Conformity assessment procedures are assigned according to this current differentiation (27).

Finally, IVDD regulates the market authorization of medical devices intended for in vitro usage, such as reagents, reagent products, calibrators, control materials, kits, instruments, apparatuses, equipment, or systems, which are used alone or in combination. Different conformity assessment procedures are applied to groups of devices based on lists, which are annexed to the directive. Devices are assigned to List A or List B or are subject to specific regulations (e.g., for performance evaluation or self-testing devices) (28).

In response to several scandals in the field of medical devices in the recent past (e.g., prefilled silicone breast implants manufactured by Poly Implants Prosthèse [PIP] (6) and hip implants manufactured by DePuy) (7), the European Commission issued two proposals on medical device regulation on 26 September 2012 and recommendations on the audits and assessments performed by notified bodies in the field of medical devices on 24 September 2013. The first proposal modifies both the MDD and the AIMDD to directly categorize active implantable devices into class III, where they would fall under de facto due to their risk potential (31). The second proposal calls for in vitro diagnostic devices to be classified into four classes according to their risk level, which necessitates different conformity assessment procedures (32).

Classification of Medical Devices According to Financial Aspects. The OECD provides within its framework "System of Health Accounts" a systematic description of financial flows concerning the consumption of healthcare goods and services. The aim of the international classification for health accounts (ICHA) is to describe healthcare systems from an expenditure perspective. One essential dimension in this classification is that of healthcare functions (ICHA-HC), which groups healthcare goods and ser- vices based on their purpose. Medical devices are assigned to different healthcare functions, for example therapeutic appliances and other medical goods (HC.5.2), which include devices such as glasses (HC.5.2.1), hearing aids (HC.5.2.2), as well as orthotic devices and other orthopedic appliances (HC.5.2.3) (33).

Sorenson and Kanavos (34) also developed a categorization of medical devices according to financial aspects, taking into account procurement policies across a sample of five European countries (England, France, Germany, Italy, Spain). They explored how such practices interact with coverage and reimbursement approaches. They used Busse's (35) three categories of devices - medical aids, implantable artificial body parts, and medical devices for the assistance of medical professionalsto explain the relationships between actors of procurement and purchasing interactions.

Global Medical Device Nomenclature (GMDN). The GMDN was developed as an international coding system for medical devices based on different existing nomenclatures. These included the Classification Names for Medical Devices (CNMD) used in the United States, the European Diagnostic Manufacturers Association (EDMA) in vitro product classification, ISO 9999 Technical Aids for Disabled Persons Classification (international use), the Japanese Medical Device Nomenclature (JFMDA), the Norsk Klassifisering Koding and Nomenklatur (NKKN), and the Universal Medical Device Nomenclature System (UMDNS) (36). The aim was to provide a common method for unambiguously describing and identifying medical devices, to be used by relevant regulatory authorities, healthcare providers, manufacturers, suppliers, and assessment bodies (37). Its structure is based on three levels: device category, generic device group, and device type (37).

\section{Developing a Taxonomic Model}

Relevant elements from the identified classification schemes were combined to form a wider taxonomic model that follows the logic of HTA for decision making on value and coverage (Figure 1). While the GMDN seems best suited for product identification in the context of market approval, vigilance reporting and recall systems, it might be inappropriate for the development of a device-taxonomy following the logic of HTA. Thus, to systematically develop the aforementioned taxonomy, characteristics of the EU Directives and the OECD Classification of Health Care Functions were primarily used. Results in the form of a $9 \times 6$ matrix are presented in Figure 1.

The nine rows show the categories included in the three Directives on medical devices, which are used to determine authorization processes, incorporating elements of risk and functionality of device types. According to the Directive 93/42/EEC, technologies that are neither an active implantable medical device (IV) nor an in vitro diagnostic medical device (V-VIII) 


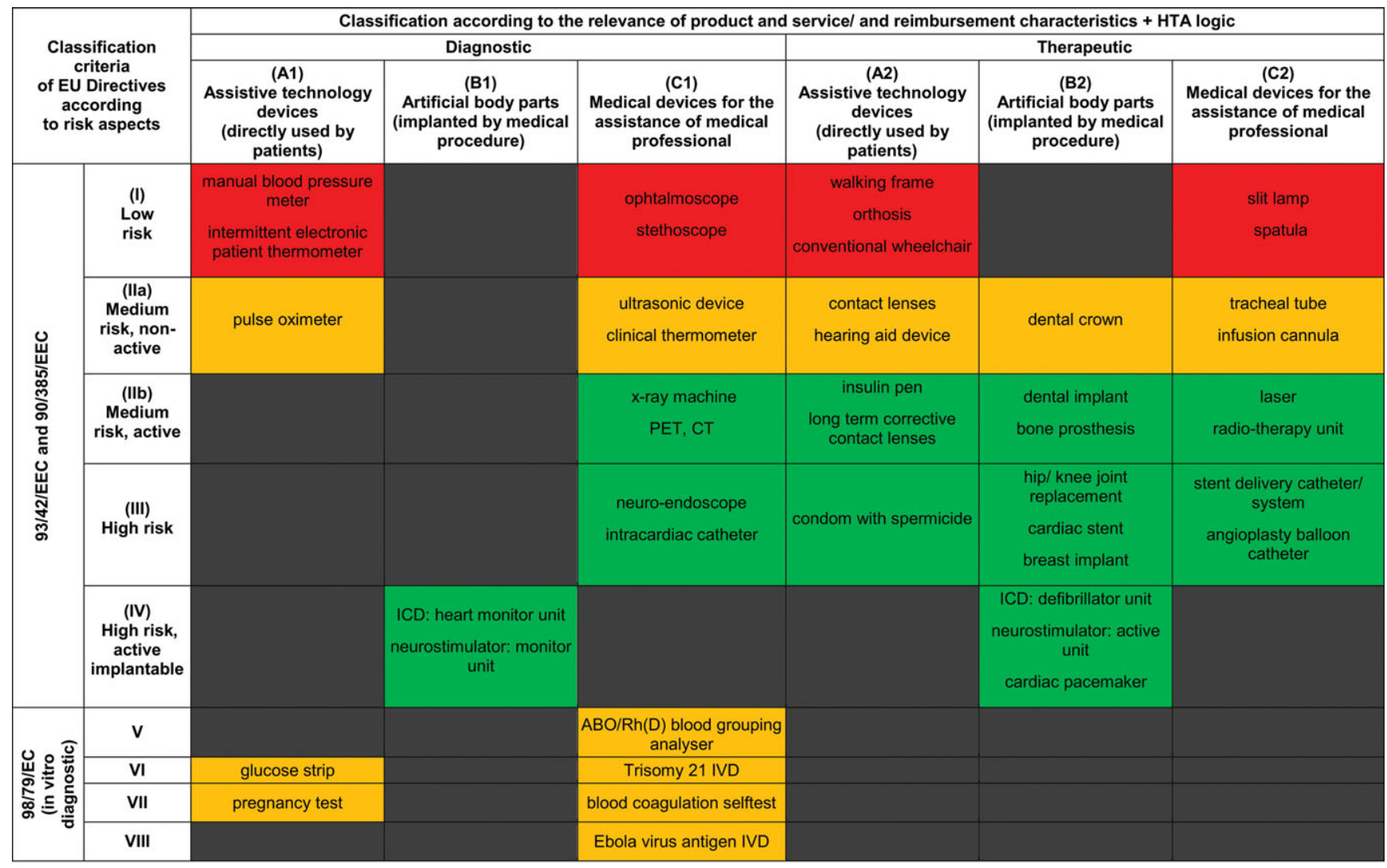

Figure 1. Taxonomic model to classify medical devices according to their HTA relevance.

are integrated in one of four classes. While medical devices in class I are associated with low potential risk, medium risk is attributed to classes IIa (nonactive) and IIb (active). Class III devices are considered high-risk while the risk associated with active implantable devices (IV) falling under Directive 90/385/EEC is deemed particularly high. Cells numbered from $\mathrm{V}$ to VIII include products classified according to the criteria of Directive 98/79/EC on in vitro diagnostic medical devices. Risk, if relevant to patient safety, is a dimension for HTA to be balanced with the patient-relevant benefit. As HTA for medical devices with high-risk potential is more important due to risks for patients and user arising from their use, a high-risk potential thus can also guide the selection process of devices that should be evaluated.

The six columns of the matrix consider whether the medical device (i) is directly used by patients (A1/ A2), (ii) constitutes part of a specific procedure (for which a device is decisive) (B1/ B2), or (iii) can be used for a variety of procedures $(\mathrm{C} 1 / \mathrm{C} 2)$. This differentiation is based on procurement/ reimbursement activities used in the OECD Classification of Health Care Functions (ICHA-HC) and in previous work by Busse (35) and Perleth et al. (38). Assistive technology devices (A1/ A2) are products dispensed to and used by patients to compensate, maintain, or improve their functional capabilities. These include class HC.5.2 (therapeutic appliances and medical goods) from the OECD ICHA-HC. For HTA purposes, these technologies are the ones closest to pharmaceuticals, at least those with a therapeutic objective. Artificial body parts (B1/ B2) are implanted by medical procedures and are largely related to class HC.1 (Curative care) in the OECD ICHA-HC. More precisely, these technologies encompass "medical procedures" in which the device is an important component. Medical devices for the assistance of medical professionals $(\mathrm{C} 1 / \mathrm{C} 2)$ include classes HC.4.1 (Laboratory services) and HC.4.2 (Imaging services) of the OECD ICHA-HC. A relevant issue for HTA is that these technologies can be used in a variety of ways (by indication, by body part, etc.). Furthermore, the columns allow for a separation of the aforementioned functions based on the diagnostic or therapeutic use of devices.

We considered the relevance of different device categories in regard to HTA to be considerably variable, ranging from high to low. For taxonomic positions with low relevance, the necessity of conducting an HTA in the first place should be discussed under consideration of efficient resource use. We propose an initial, theoretical color-coding in the matrix (its plausibility will be tested in a subsequent step, see discussion below). High HTArelevance of different device categories is highlighted green, low relevance red. Cells marked yellow include groups where relevance is context-sensitive. Grey fields denote cells for which no medical devices were identified ( 29 of the 54 cells). The color coding of the taxonomy could be used to inform decision makers when an HTA should be undertaken. The extent to which 
assessment tools should be tailored to achieve best results depending on different categories of medical devices is discussed below.

\section{DISCUSSION}

The developed taxonomy combines different device classification schemes that have been used for different purposes. Its aim is to provide decision makers (and at a later stage, assessors) with a tool enabling them to consider device risk in more detail across more than one dimension. The placement of device groups in the model cells provides decision support on the necessity of conducting a full HTA in the first place. It additionally illustrates that choosing to assess a given device may be inefficient in some cases (e.g., cell A1/ I) or particularly complex in others (e.g., cell B1/ IV especially in combination with cell B2/ IV). Furthermore, that different taxonomic positions may merit separate additional considerations both at the conceptual stage of assessment as well as in the methodological approach adopted. The model can thus provide helpful insight during the operationalization of the research question along the lines of the PICO model and the development of the HTA protocol (e.g., on relative taxonomic position of comparators, spectrum of relevant outcomes, study design etc.).

The three EU Directives on medical devices used in the licensing process focus on elements of risk and functionality. Device risk is highly important for patient safety and is therefore one of the required domains both in pre- and postmarket evaluation processes. From the HTA-perspective, it can also guide topic prioritization processes, as policy makers should pay particular attention to high-risk devices (39) (e.g., implantable or surgically invasive devices). Furthermore, risk classification in the MDD also depends on the duration of patient-device contact (transient: $<60 \mathrm{~min}$, short term: continuous use $60 \mathrm{~min}-$ 30 days, long term: continuous use $>30$ days). This might be a significant detail to consider during assessment, as duration of contact can play an important role in data collection (e.g., dental implants) and thus required duration of studies considered acceptable as evidence. Generally, longer contact duration is associated with a higher risk for patients and, therefore, a higher need for HTA.

The separation of assistive technology devices, artificial body parts and devices for the assistance of medical professionals is an essential part of the matrix from an HTA viewpoint. While assistive technology devices are products directly used by patients and can in most cases be assessed "alone", artificial body parts and medical devices for the assistance of medical professionals are both used within procedures and are subject to device-operator interaction. Thus, effectiveness outcomes are also dependent on the process or its operator. In addition, they often need to be compared with the current standard procedures. Therefore, assessment tools need to be able to account for those dimensions. Furthermore, how certain procedures are to be assessed may depend on whether they are a part of a wider treatment plan under a physician's care (40).

The taxonomic model further separates diagnostic from therapeutic devices, thus recognizing that different methodological approaches need to be adopted depending on the nature of the device. This is in agreement with international reference models, such as the HTA Core Model ${ }^{\mathrm{TM}}$ developed by EUnetHTA. The Model's second version was published in late 2013 and comes in different iterations for diagnostic and therapeutic technologies, the former explicitly addressing issues of clinical utility and clinical validity of diagnostic tests (41). Furthermore, NICE in England has a separate program for diagnostic technologies, incorporating appropriate methods (42). As a rule, it is important to recognize that for some devices, it is challenging to separate the value of the diagnostic test per se from the value of improved outcomes as a result of subsequent treatment. We argue that a potential approach for devices with both diagnostic and therapeutic functions (cell combination B1/ IV and B2/ IV) would be for the two components to be considered separately.

In its presented form, the matrix model is not without limitations. The color-coded relevance of HTA for each device category currently relies on several assumptions. Thus, to test the model's plausibility, a subsequent step in our work entails filtering available HTA reports of European institutions and assigning taxonomic positions to devices assessed. Furthermore, the approach toward risk used in the EU regulation and transferred to the technology is product-specific, thus ignoring the contribution of patient characteristics (such as severity of condition) toward the overall risk of the intervention. Finally, as the current taxonomy is focused on the European context, it is possible to strengthen the model by including classification criteria from other contexts, such as those of the FDA.

Issues that pertain to individual device groups, such as battery life for active implantable devices or learning curves where device-operator interaction applies, may pose additional challenges that need to be recognized and be accounted for to produce robust and representative assessments. Therein lies one of the intentions of the presented model, namely to fuel the debate on when and to what extent a differentiated approach is appropriate and necessary. Thus, the taxonomy does not elaborate on individual elements at this stage but rather draws attention to the categorical position of each device and its core properties to point in the direction of what device-specific elements may be. The next step in our work will include the establishment of basic methodologies per taxonomic position to explicitly consider particularities of different categories of medical devices.

\section{CONCLUSIONS}

Existing medical device classifications are not sufficient for the purposes of HTA evaluation as they are based on different underlying logics. Furthermore, general HTA methodology, while it 
may incorporate important considerations such as the therapeutic versus diagnostic nature of devices, does not consistently account for device-specific elements such as device-operator interaction, duration of patient-device contact, level of device activity, or combined diagnostic/therapeutic functionality. The new developed taxonomy combines the two worlds and add further considerations to provide a tool enabling decision makers to consider device risk in more detail across more than one dimension; it can thus provide decision support on the necessity of conducting a full HTA in the first place. The taxonomy thus may be considered an important step toward advancing the debate on HTA/economic evaluation by grouping medical devices according to their relevance for HTA activities. Ongoing steps include testing taxonomy's plausibility by filtering available HTA reports produced by European institutions to assign medical devices a taxonomic position, and establishing basic methodologies (e.g., study design requirements) in HTA, which explicitly consider peculiarities of different categories of medical devices.

\section{ACKNOWLEDGEMENTS}

We are grateful to Sabine Fuchs, Britta Olberg and Susanne Felgner for their constructive comments.

\section{CONFLITS OF INTEREST}

This paper is the result of research that was funded through the European Commission's FP7 Cooperation Work Programme: Health (Grant Agreement Number 305983; acronym ADVANCE_HTA). The European Commission is not responsible for the content of the paper. The authors declare no further conflict of interest in conjunction with this work.

\section{REFERENCES}

1. European Parliament and Council of the European Union. Directive 2007/47/EC of the European Parliament and of the Council of 5 September 2007 amending Council Directive 90/385/EEC on the approximation of the laws of the Member States relating to active implantable medical devices, Council Directive 93/42/EEC concerning medical devices and Directive 98/8/EC concerning the placing of biocidal products on the market. http://ec.europa.eu/consumers/sectors/ medical-devices/files/revision_docs/2007-47-en_en.pdf (accessed June 3, 2015).

2. Robinson JC. Value-based purchasing for medical devices. Health Aff (Millwood). 2008;27:1523-1531.

3. Cutler DM. The lifetime costs and benefits of medical technology. $J$ Health Econ. 2007;26:1081-1100.

4. Sorenson C, Drummond M, Wilkinson G. Use of innovation payments to encourage the adoption of new medical technologies in the English NHS. Health Policy Technol. 2013;2:168-173.

5. Horton R. Offline: A serious regulatory failure, with urgent implications. Lancet. 2012;379:106.

6. Heneghan C. The saga of Poly Implant Prosthèse breast implants. $B M J$. 2012;344:e306.

7. Cohen D. How safe are metal-on-metal hip implants? BMJ. 2012;344:e1410.
8. Maisel WH, Sweeney MO, Stevenson WG, Ellison KE, Epstein LM. Recalls and safety alerts involving pacemakers and implantable cardioverter-defibrillator generators. JAMA. 2001;286:793-799.

9. Hillman BJ. Government health policy and the diffusion of new medical devices. Health Serv Res. 1986;21:681-711.

10. Borgonovi E, Busse R, Kanavos P. Financing medical devices in Europe: Current trends and perspectives for research. Eurohealth. 2008;14:1-3.

11. Kramer DB, Xu S, Kesselheim AS. Regulation of medical devices in the United States and the European Union. NEngl J Med. 2012;366:848-855.

12. Kaplan AV, Baim DS, Smith JJ, et al. Medical device development: From prototype to regulatory approval. Circulation. 2004;109:3068-3072.

13. Zentner A, Busse R. Bewertung von Arzneimitteln - Wie gehen andere Länder vor? Gesundheit Ges Wiss 2011;11:25-34.

14. Draborg E, Gyrd-Hansen D, Poulsen PB, et al. International comparison of the definition and the practical application of health technology assessment. Int J Technol Assess Health Care. 2005;21:89-95.

15. Migliore A, Ratti M, Cerbo M, et al. Health technology assessment: Managing the introduction and use of medical devices in clinical practice in Italy. Expert Rev Med Devices. 2009;6:251-257.

16. Hutton J, McGrath C, Frybourg JM, et al. Framework for describing and classifying decision-making systems using technology assessment to determine the reimbursement of health technologies (fourth hurdle systems). Int J Technol Assess Health Care. 2006;22:10-18.

17. Drummond M, Griffin A, Tarricone R. Economic evaluation for devices and drugs - same or different? Value Health. 2009;12:402-404.

18. Campillo-Artero C. A full-fledged overhaul is needed for a risk and value-based regulation of medical devices in Europe. Health Policy. 2013;113:38-44.

19. WHO. Development of medical device policies. WHO Medical device technical series. 2011. http://whqlibdoc.who.int/publications/ 2011/9789241501637_eng.pdf?ua=1 (accessed June 3, 2015).

20. Kirisits A, Redekop WK. The economic evaluation of medical devices. Appl Health Econ Health Policy. 2013;11:15-26.

21. McCulloch P, Altman DG, Campbell WB, et al. No surgical innovation without evaluation: The IDEAL recommendations. Lancet. 2009;374:1105-1112.

22. Taylor RS, Iglesias CP. Assessing the clinical and cost-effectiveness of medical devices and drugs: Are they that different? Value Health. 2009;4:404-406.

23. Hulstaerta F, Neyta M, Vinck I, et al. Pre-market clinical evaluations of innovative high-risk medical devices in Europe. Int J Technol Assess Health Care. 2012;28:278-284.

24. Ergina PL, Cook JA, Blazeby JM, et al. Challenges in evaluating surgical innovation Lancet. 2009;374:1097-1104.

25. Bossuyt PM, Irwig L, Craig J, et al. Comparative accuracy: Assessing new tests against existing diagnostic pathways. BMJ. 2006;332;10891092.

26. European Parliament and Council of the European Union. Council Directive 93/42/EEC of 14 June 1993 concerning medical devices. http://eur-lex.europa.eu/legal-content/EN/TXT/PDF/?uri= CELEX:31993L0042\& from=EN (accessed June 3, 2015).

27. European Parliament and Council of the European Union. Council Directive 90/385/EEC of 20 June 1990 on the approximation of the laws of Member states relating to active implantable medical devices. http://eur-lex.europa.eu/legal-content/EN/TXT/PDF/?uri= CELEX:31990L0385\&from=EN (accessed June 3, 2015).

28. European Parliament and Council of the European Union. Council Directive 98/79/EC of 27 October 1998 on in vitro diagnostic medical devices. http://eur-lex.europa.eu/legal-content/EN/TXT/PDF/?uri= CELEX:31998L0079\&from=EN (accessed June 3, 2015).

29. Altenstetter C. EU and member state medical devices regulation. Int $J$ Technol Assess Health Care. 2003;19:228-248. 
30. Altenstetter $\mathrm{C}$. Regulating health care technologies and medical supplies in the European Economic Area. Health Policy. 1996;35:33-52.

31. European Commission 2012. Proposal for a regulation of the European parliament and of the council on medical devices, and amending Directive 2001/83/EC, regulation (EC) No 178/2002 and regulation (EC) No 1223/2009. http://ec.europa.eu/health/medicaldevices/files/revision_docs/proposal_2012_542_en.pdf (accessed June 3, 2015).

32. European Commission 2012. Proposal for a regulation of the European parliament and of the council on in vitro diagnostic medical devices. http://ec.europa.eu/health/medical-devices/files/revision_docs/ proposal_2012_541_en.pdf (accessed June 3, 2015).

33. OECD, Eurostat, WHO. A system of Health Accounts. OECD Publishing. 2011. http://www.who.int/nha/sha_revision/sha_2011_final1.pdf (accessed June 3, 2015).

34. Sorenson C, Kanavos P. Medical technology procurement in Europe: A cross-country comparison of current practice and policy. Health Policy. 2011;100:45-50.

35. Busse R. Decision-making: The link between reference pricing and procurement. Brussels. 15.10.2008. http://www.mig.tu-berlin.de/ fileadmin/a38331600/2008.lectures/Brussel_2008.10.15_rb_EHTI.pdf (accessed June 3, 2015).
36. Anand K, Saini SK, Singh BK, et al. Global medical device nomenclature: The concept for reducing device-related medical errors. $J$ Young Pharm. 2010;2:403-409.

37. GMDN Agency. The global medical device nomenclature. 2012. https://www.gmdnagency.org/ (accessed June 3, 2015).

38. Perleth M, Busse R, Schwartz FW. Regulation of health-related technologies in Germany. Health Policy. 1999;46:105-126.

39. Krüger LJ, Wild C. Evidence requirements for the authorization and reimbursement of high-risk medical devices in the USA, Europe, Australia and Canada: An analysis of seven highrisk medical devices. Vienna: Ludwig Boltzmann Institut fuer Health Technology Assessment (LBIHTA); HTA-Projektbericht 73. 2013

40. Olberg B, Perleth M, Busse R. The new regulation to investigate potentially beneficial diagnostic and therapeutic methods in Germany: Up to international standard? Health Policy. 2014;117:135145.

41. EUnetHTA. Joint Action 2, Work Package 8. HTA Core Model ${ }^{\circledR}$ version 2.0; 2013. http://www.corehta.info/BrowseModel.aspx (accessed June 3, 2015).

42. Adrian Newland, Chair, NICE Diagnostics Advisory Committee. NICE diagnostics assessment programme. Ann R Coll Surg. 2011;93:412-413. 